\title{
L'hémoglobine glyquée : un marqueur de choix en Toxicologie Médico-Légale
}

\section{Glycated haemoglobin : a marker of interest in forensic Medicine}

Jean-Pierre GOULLÉ ${ }^{(1)}$, Christian LACROIX ${ }^{(1)}$, Daniel BOUIGE ${ }^{(2)}$

(1) Laboratoire de Pharmacocinétique et de Toxicologie Cliniques, Groupe Hospitalier, B.P. 24 - 76083 LE HAVRE Cedex - Tél : 0232733223 - Fax : 0232733238

(2) Laboratoire de Biochimie, Groupe Hospitalier, B.P. 24 - 76083 LE HAVRE Cedex Tél : 0232733370 - Fax : 0232733372

*Auteur à qui adresser la correspondance : Dr Jean-Pierre GOULLÉ, Laboratoire de Pharmacocinétique et de Toxicologie Cliniques, Centre Hospitalier Jacques Monod - B.P. 2476083 LE HAVRE Tél : 0232733223 - Fax : 0232733238

(Reçu le 25 juillet 2000 ; accepté le 17 août 2000)

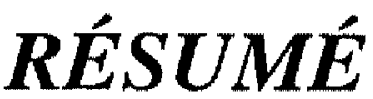

Le dosage de l'hếmoglobine glyquée $\left(H b A_{l}\right.$ ) est considéré comme un élément objectif du contrôle de l'équilibre glycémique du malade diabétique. Ce paramètre caractérise toute fixation non enzymatique de glucose sur l'hémoglobine. Il est le reflet de la glycémie moyenne des 60 derniers jours et complète donc utilement les mesures ponctuelles de glycémie. C'est en quelque sorte "le cheveu du glucose". Nous avons étudié la conservation de l' $H b A_{I C}$ à $+4^{\circ} \mathrm{C}$ au cours du temps avec différents anticoagulants ou conservateurs ( 3 mois, 6 mois, $l$ an). 106 dosages ont été réalisés sur un ensemble CLHP dédié à l'analyse semi-automatique de l'hémoglobine $A_{I C}$ (Biorad). La technique a été appliquée à des cas médico-légaux. Les résultats montrent que la conservation est bonne jusqu'à 3 mois dans les échantillons prélevés sur fluorure, 6 mois dans ceux prélevés sur tube sec ou sur tube hépariné. Chez des sujets non diabétiques, les valeurs de référence obtenues sur les prélèvements médico-légaux sont identiques à celles de témoins vivants (3,5-6,25\% d'hémoglobine totale). Les résultats positifs ont tous été confirmés par l'enquête médicale. La technique a été appliquée avec succès à quatre observations médico-légales. Son augmentation permet, en cas d'acétonémie élevée d'exclure une intoxication par l'acétone ou l'isopropanol mais également de différencier l'acidocétose alcoolique ou de jeûne de l'acidocétose diabétique. L'hémoglobine glyquée constitue un marqueur de choix du diabète dont l'interprétation est valide en post-mortem.

MOTS-CLÉS

Acétone, hémoglobine glyquée, diabète, cas mortels.

\section{SUMMARY}

The measurement of glycated hemoglobin $\left(H b A_{1 C}\right)$ is considered to be an objective element in the control of the glyce. mic equilibrium of a diabetic patient. This parameter characterises every non enzymatic fuxation of glucose on hemoglobin. It is a useful complement of the punctual glycemia measurements since it reflects the mean glycemia of the last 60 days. We studied the conservation of $\mathrm{HbA}_{1 \mathrm{C}}$ at $+4^{\circ} \mathrm{C}$ as a function of time with different anticoagulants and preservatives ( 3 months, 6 months, 1 year). 106 measurements were performed on an HPLC method dedicated to the semiautomatic analysis of hemoglobin $A_{I C}$ (Biorad). This method was applied to forensic cases. Conservation is good for as long as 3 months in samples collected with fluoride and 6 months in samples collected in a dry or in a heparinized tube. In non diabetic subjects reference values obtained on forensic samples were identical to those of living controls (3,5-6,25 \% of total hemoglobin). Positive results were all confirmed by a medical enquiry. This method was successfully applied to four forensic observations. Increased $\mathrm{HbAIC}$ allows to rule out an intoxication by acetone or isopropanol in cases of increased acetonemia and likewise to differentiate alcoholic or fasting acid ketosis from the diabetic one. Glycated hemoglobin is thus the marker of choice of diabetes, its interpretation being valid post mortem.

\section{KEY-WORDS}

Acetone, glycated hemoglobin, diabetes, fatal cases. 


\section{Introduction}

Le diabète est une maladie fréquente, sa prévalence est estimée à environ $3 \%$ de la population générale. On distingue différentes formes de diabète : le diabète non insulino-dépendant (DNID) représente à lui seul les $2 / 3$ des cas soit $2 \%$ de la population, le diabète de type 1 ou insulino-dépendant (DID) touche $0,25 \%$ de la population genérale, les autres formes de diabète étant plus rares : diabète lipo-atrophique ou diabète insulino-résistant ; diabète secondaire à des maladies chromosomiques, génétiques, dégénératives, endocriniennes ou autres, dû à une insuline anormale ; diabète de la grossesse. Les décès dus aux complications du diabète sont toujours de diagnostic difficile : absence de signe autopsique, la glycémie après le décès n'est pas interprétable ; celle-ci pouvant être nulle ou considérablement augmentée dans les minutes qui suivent le décès. Les paramètres biochimiques classiques ne sont pas utilisables étant également modifiés instantanément après le décès : $\mathrm{pH}$, bicarbonates, lactates, potassium. L'acétone constitue le seul marqueur post-mortem valide comme cela a été montré par plusieurs auteurs $(1,2$, $3,4,5)$. En effet, cette molécule se conserve relativement bien dans le cadavre, aucune production n'y est constatée au moins 5 jours après le décès (4). Cependant des valeurs très élevées d'acétonémie peuvent avoir des origines variées : acidocétose du jeûne, intoxication par l'acétone ou l'isopropanol, acidocétose diabétique, acidocétose alcoolique. Hormis les cas exceptionnels d'intoxication par l'acétone ou l'isopropanol, toute élévation de l'acétonémie est le témoin d'une déviation métabolique dont l'origine ne peut être précisée. Le décès du diabétique est probablement lié davantage à des concentrations anormales de glucose, très faibles dans les cellules et très élevées dans le sang, qu'aux valeurs élevées de l'acétonémie (6). Le décès par acidocétose et en l'absence d'autre cause chez l'alcoolique chronique est vraisemblablement lié à un emballement de la lipolyse en période d'abstinence d'alcool et après plus de 24 heures de jeûne (1).

L'objectif de ce travail est d'apprécier la pertinence et les conditions de la détermination de l'hémoglobine glyquée en médecine légale, en particulier de préciser la nature de la déviation métabolique chez les sujets décédés. L'hémoglobine glyquée caractérise toute fixation non enzymatique du glucose sur l'hémoglobine. L'hémoglobine $A_{1 C}\left(\mathrm{HbA}_{1 \mathrm{C}}\right)$ est formée par la fixation de glucose sur l'extrémité $N$-terminale des chaînes $\beta$ de $1^{\prime} \mathrm{HbA}_{1}$ : Cette fixation est d'abord labile puis devient stable et est proportionnelle à la glycémie. La teneur en hémoglobine $A_{1 C}$ stable est le reflet de la glycémie moyenne des 60 derniers jours précédant le prélèvement. Les valeurs normales s'échelonnent de 3,5 à $6,25 \%$ d'hémoglobine totale. L'HbA ${ }_{1 c}$ constitue un élément objectif du contrôle de l'équilibre glycémique chez le diabétique. Au cours du traitement, sa valeur doit être maintenue à une valeur inférieure à $7 \%$, correspondant à une glycémie moyenne de $1,47 \mathrm{~g} / \mathrm{l}$ selon la formule de Nathan (7) : glycémie moyenne estimée $=0,33 \times \mathrm{HbA}_{1 \mathrm{C}}-0,86 \mathrm{~g} / \mathrm{l}$. Seules les techniques dosant spécifiquement l'HbA $\mathrm{A}_{1 \mathrm{C}}$ sont recommandées : chromatographie d'échange d'ions, minicolonnes, chromatographie liquide haute performance (CLHP), chromatographie liquide basse pression (CLBP), électrophorèse, méthodes immunologiques (8). S'il n'existe pas à ce jour de méthode de référence, la commission "Protéines glyquées" de la Société Française de Biologie Clinique (Président P. Gillery) considère que les techniques de CLHP apparaissent les plus performantes (8). C'est la méthode que nous avons retenue en raison de l'existence d'échantillons altérés, mais aussi de la nécessité d'une identification formelle de $\mathrm{l}^{\prime} \mathrm{HbA}_{\mathrm{IC}}$ des autres fractions de l'hémoglobine, critère indispensable en médecine légale.

\section{Matériel et méthode}

\section{Matériel}

Il s'agit d'un ensemble de chromatographie liquide haute pression (CLPH) de type Diamat (Biorad, Ivry sur Seine, France) dédié à l'analyse semi-automatique de l' $\mathrm{HbA}_{1 \mathrm{C}}$. Les différentes fractions de l'hémoglobine sont séparées sur une colonne échangeuse de cations (résine Poly CAT) à $30^{\circ} \mathrm{C}$, par un gradient en 3 paliers de phases mobiles de force ionique croissante fournies par l'industriel. Les mesures sont réalisées en bichromatisme à 415 et $690 \mathrm{~nm}$ après dilution du sang $(4 \mu \mathrm{l})$ dans un tampon hémolysant fourni avec les réactifs $(1 \mathrm{ml})$, puis incubation 30 minutes à $37^{\circ} \mathrm{C}$. Cette opération permet d'éliminer l'hémoglobine glyquée labile. Un standard lyophilisé est traité dans les mêmes conditions. Les échantillons sont ensuite placés sur un carrousel où ils sont prélevés automatiquement. La durée d'une analyse est de $10 \mathrm{~min}$. A l'issue de la séparation, le chromatogramme est édité et l' $\mathrm{HbA}_{1 \mathrm{C}}$ automatiquement calculée. Les performances du système données par le fabricant sont les suivantes (9) :

- répétabilité :

- sujets normaux $(\mathrm{n}=20)$ : pour $\mathrm{HbA}_{\mathrm{IC}} 5 \%$ - $\mathrm{CV}<2,3 \%$ - sujets diabétiques $(\mathrm{n}=20)$ : pour $\mathrm{HbA}_{1 \mathrm{C}} 10 \%-\mathrm{CV}<$ $1,5 \%$ 
- reproductibilité :

- sujets normaux $(\mathrm{n}=20)$ : pour $\mathrm{HbA}_{1 \mathrm{C}} 5 \%-\mathrm{CV}<3,1 \%$

- sujets diabétiques $(\mathrm{n}=20)$ : pour $\mathrm{HbA}_{1 \mathrm{C}} 10 \%-\mathrm{CV}<$ $1,2 \%$

- récupération d'ajouts : pour des valeurs d' $\mathrm{HbA}_{1 \mathrm{C}}$ comprises entre 8,7 et $18,6 \%$ le pourcentage de récupération varie de 96,8 à $100,0 \%$.

- L'interférence éventuelle constituée par la fraction $\mathrm{HbA}_{1 \mathrm{C}}$ labile qui peut conduire à des résultats artificiel-

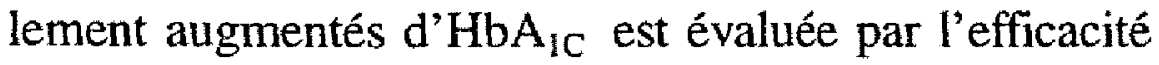
du déplacement de la base de Schiff : l'incubation de sang provenant d'un sujet normal et d'un malade diabétique, 5 heures à $37^{\circ} \mathrm{C}$ en présence de glucose à 2,5 et $5,0 \mathrm{~g} / \mathrm{l}$, montre que la base de Schiff est complètement déplacée lors du prétraitement de l'échantillon.

- Prélèvements lipémiques : la présence de lipoprotéines est sans influence sur le résultat d' $\mathrm{HbA}_{1 \mathrm{C}}$.

- Interférences dues à la présence d'hémoglobines anormales : hémoglobine $\mathrm{F}$ (foetale) : pas d'interférence jusqu'à $5 \%, \mathrm{HbA}_{2}$ (thalassémie majeure) : pas d'interférence jusqu'à $1,5 \%$, d'hémoglobine $\mathrm{S}$ (drépanocytose) et d'hémoglobine $\mathrm{C}$ (anémie à hématies falciformes) : pas d'interférence dans les formes hétérozygotes en revanche dans les formes homozygotes un autre critère doit être utilisé en raison de l'absence d'hémoglobine A.

\section{Population étudiée}

Il s'agit de prélèvements fluorés destinés à la mesure d'alcoolémies médico-légales ou d'échantillons fluorés, héparinés, sur EDTA ou de type "sec", provenant d'autopsies en vue de leur expertise toxicologique. Une étude de conservation à $+4^{\circ} \mathrm{C}$ est réalisée sur les différents types de prélèvements après différentes périodes de stockage ( 3 mois, 6 mois, 1 an). La technique a été appliquée à quatre cas médico-légaux présentant des acétonémies élevées, afin de préciser l'origine de la déviation métabolique.

\section{Résultats}

\section{Étude de conservation}

- sur fluorure à $0,25 \%(p / v)$ : prélèvements d'alcoolémies médico-légales

Les résultats sont reportés dans le tableau I. Nous constatons que la conservation à $+4^{\circ} \mathrm{C}$ est bonne pendant une période minimale de 3 mois. Comme le montre la figure 1, l'hémoglobine $A_{1 C}$ est bien séparée des autres fractions. Après 6 mois la dénaturation de l'échantillon affecte la résolution chromatographique et ne permet plus de séparer l' $\mathrm{HbA}_{\mathrm{IC}}$ de l'hémoglobine totale (figure 2).

Figure 1 : Tracé montrant la séparation de l' $\mathrm{Hb} \mathrm{A}_{0}$ et de l'hémoglobine glyquée ou $\mathrm{HbA}$.
Tableau I: Conservation de l' $H b A_{l C} \grave{a}+4^{\circ} \mathrm{C}$ sur tube fluoré après 3, 6, 12 mois.

\begin{tabular}{|c|c|c|}
\hline Durée & $\mathbf{n}$ & Tracés interprétables \\
\hline 3 mois & 10 & 9 \\
\hline 6 mois & 10 & 3 \\
\hline 1 an & 10 & 0 \\
\hline
\end{tabular}
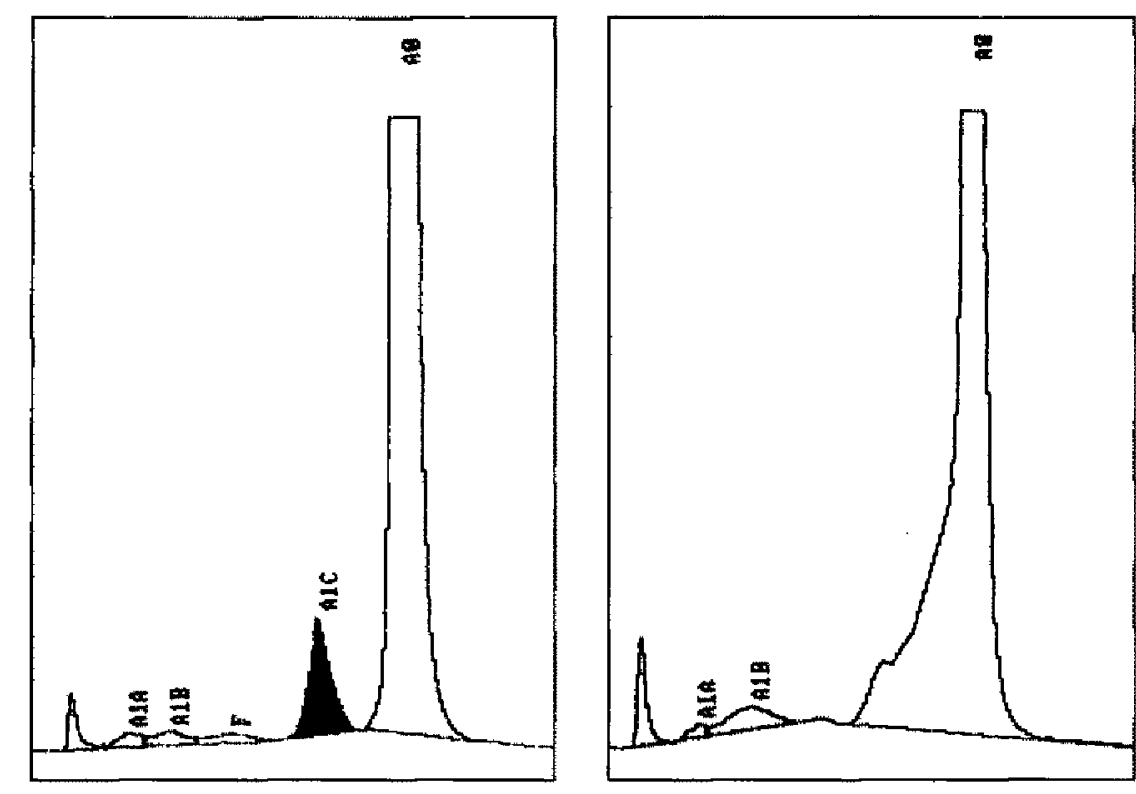

Figure 2 : Tracé montrant un pic $H b A_{\text {Id }}$ dénaturée interférant avec la détermination de l'hémoglobine glyquée ou $\mathrm{HbA}_{I C}$.

\section{- sur tube sec : prélèvements autopsiques}

Les résultats du tableau II montrent que la conservation $\grave{a}+4^{\circ} \mathrm{C}$ pendant 1 an est bonne.

- sur héparine ou sur EDTA : prélèvements autopsiques

Les résultats du tableau III, même s'ils sont en petit nombre $(n=8)$ semblent indiquer que l'EDTA et l'héparine conviennent.

\section{Comparaison de l' $\mathrm{HbA}_{1 \mathrm{C}}$ sur des paires de prélèvements autopsiques}

Pour sept autopsies pour lesquelles la détermination de l' $\mathrm{HbA}_{1 \mathrm{C}}$ a été réalisée au maximum un mois après l'autopsie, nous avons pu comparer les résultats (Tableau IV) obtenus à partir de sang prélevé à la fois sans conservateur et avec un additif (fluorure, héparinate), nous ne constatons pas de différence a priori significative.

\section{Sujets présentant une $\mathrm{HbA}_{1 \mathrm{C}}$ élevée $(6,25 \%)$}

Au cours de l'étude de conservation, cinq sujets ont présenté des valeurs anormalement élevées d'HbA $\mathrm{H}_{\mathrm{iC}}$ (Tableau V), chez lesquels le diagnostic de diabète a pu être confirmé. 
Tableau II : Conservation de l'HbA ${ }_{1 C} \grave{a}+4^{\circ} \mathrm{C}$ sur tube sec après 3, 6, 12 mois.

\begin{tabular}{|c|c|c|}
\hline Durée & $\mathbf{n}$ & Tracés interprétables \\
\hline 3 mois & $9^{*}$ & 8 \\
\hline 6 mois & 7 & 7 \\
\hline 1 an & $11^{* *}$ & 10 \\
\hline
\end{tabular}

*1 échantillon : prélèvement sur cadavre de nouveau-né $\mathrm{Hb}$ fótale ( $\mathrm{Hb}$ F) **1 échantillon absence d'hémoglobine détectable

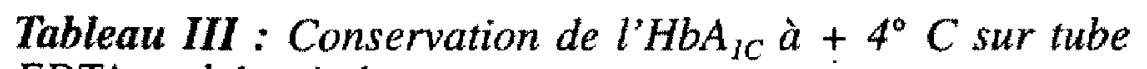
EDTA ou hépariné.

\begin{tabular}{|c|c|c|}
\hline 25iree & 7. & Tracés interprétables \\
\hline 3 mois & $\begin{array}{c}2 \text { héparine } \\
1 \text { EDTA }\end{array}$ & $\begin{array}{l}1 \\
1\end{array}$ \\
\hline 6 mois & $\begin{array}{l}2 \text { héparine* } \\
1 \text { EDTA }\end{array}$ & $\begin{array}{l}1 \\
1\end{array}$ \\
\hline 1 an & $\begin{array}{l}1 \text { héparine } \\
\text { I EDTA }\end{array}$ & $\begin{array}{l}1 \\
1\end{array}$ \\
\hline
\end{tabular}

*1 échantillon $\mathrm{Hb}$ en quantité insuffisante

Tableau IV : Comparaison des résultats $l^{\prime} H b A_{l C}$ sur des paires de prélèvement.

\begin{tabular}{|c|c|c|}
\hline Tube see & Tube fluore & Tube héparine \\
\hline $4,7 \%$ & $4,7 \%$ & \\
\hline $5,8 \%$ & $5,8 \%$ & \\
\hline $12,4 \%$ & $12,0 \%$ & \\
\hline $4,6 \%$ & $4,5 \%$ & \\
\hline $4,9 \%$ & $4,7 \%$ & \\
\hline $11,1 \%$ & & $10,5 \% *$ \\
\hline $5,9 \% * *$ & & $6,0 \% * *$ \\
\hline
\end{tabular}

* 3 mois à $+4^{\circ} \mathrm{C}$

** 6 mois à $+4^{\circ} \mathrm{C}$

Tableau $V: H b A_{I C}$ et résultats pathologiques.

\begin{tabular}{|c|c|c|}
\hline HbA & Glycemie calculee & Diagnostic \\
\hline $7,4 \%$ & $\begin{array}{c}1,60 \mathrm{~g} / 1 \\
2,00 \mathrm{à} 3,40 \mathrm{~g} /\end{array}$ & DNID \\
\hline $12,2 \%$ & $3,20 \mathrm{~g} / 1$ & DID \\
\hline $6,9 \%$ & $1,40 \mathrm{~g} / 1$ & DNID \\
\hline $10,1 \%$ & $2,50 \mathrm{~g} / 1$ & DNID \\
\hline $10,5 \%$ & $2,80 \mathrm{~g} / 1$ & DNID \\
\hline
\end{tabular}

* glycémies mesurées lors de l'hospitalisation - DNID : diabète non insulinodépendant - DID : diabète insulino-dépendant

\section{Application à des cas médico-légaux}

- Observation $N^{\circ} 1$

Il s'agit d'un homme de 55 ans, trouvé mort à son domicile, diabétique, vivant seul et ne se traitant plus selon les informations recueillies par les enquêteurs. L'autopsie ne permet pas de connaitre l'origine du décès. L'analyse toxicologique est négative (oxyde de carbone, immunoanalyse, chromatographie liquide barrette de diodes (HPLC/BD), chromatographie gazeuse - spectrométrie de masse (GC/MS), éthylène glycol, éthanol, méthanol). L'acétonémie est égale à $515 \mathrm{mg} / \mathrm{l}(\mathrm{N}<10)$ et l'isopropanolémie $150 \mathrm{mg} / \mathrm{l}$. $\mathrm{L}^{\prime} \mathrm{HbA}_{1 \mathrm{C}}$ à $17,8 \%$ révèle que la glycémie moyenne des 60 derniers jours s'établit à $5,00 \mathrm{~g} / \mathrm{l}$.

\section{- Observation $N^{\circ} 2$}

C'est un homme de 21 ans, diabétique insulino-dépendant, admis en réanimation pour coma diabétique, dans ce service il présente un arrêt cardiaque non récupérable. Le médecin légiste ne constate aucune anomalie à l'autopsie. L'analyse toxicologique est négative (oxyde de carbone, immunoanalyse, chromatographie liquide - barrette de diodes (HPLC/BD), chromatographie gazeuse - spectrométrie de masse (GC/MS), éthylène glycol, éthanol, méthanol). L'acétonémie s'établit à $405 \mathrm{mg} / \mathrm{l}(\mathrm{N}<10)$ et l' $\mathrm{HbA}_{1 \mathrm{C}}$ à $10,1 \%$ traduisant une glycémie moyenne à $2,40 \mathrm{~g} / \mathrm{l}$.

\section{- Observation $N^{\circ} 3$}

Elle concerne un homme de 47 ans, découvert mort à son domicile, très amaigri, dont le décès est imputé à une dénutrition et à une misère physiologique. L'analyse toxicologique est négative (oxyde de carbone, immunoanalyse, chromatographie liquide - barrette de diodes (HPLC/BD), chromatographie gazeuse spectrométrie de masse (GC/MS), éthylène glycol, éthanol, méthanol). L'acétonémie est égale à $259 \mathrm{mg} / \mathrm{l}$ $(\mathrm{N}<10)$. L'HbA $1 \mathrm{C}$ s'établit à $8,6 \%$ d'hémoglobine totale. Elle révèle que la glycémie moyenne des 60 derniers jours est de 2,00 g/l. L'amaigrissement récent semble donc en rapport avec l'apparition d'un diabète chez un sujet qui n'a pas consulté. Le décès est compatible avec l'une des complications d'un coma diabétique acido-cétosique.

\section{- Observation $N^{\circ} 4$}

Il s'agit d'une femme de 36 ans, suivie en milieu psychiatrique trouvée morte à son domicile, sans trace de violence, diabétique insulino-dépendante. L'autopsie ne permet pas de connaître l'origine du décès. L'analyse toxicologique est peu productive (éthanol : $0,72 \mathrm{~g} / \mathrm{l}$, méprobamate : $26 \mathrm{mg} / \mathrm{l}$, desméthyldiazepam : $0,14 \mathrm{mg} / \mathrm{l}$ ). L'acétonémie est égale à $541 \mathrm{mg} / \mathrm{l}$ (normale $<10)$. L'HbA $1 \mathrm{c}$ à $9,1 \%$, mesurée 1 an après l'autopsie sur le sang fluoré conservé à $+4^{\circ} \mathrm{C}$, révèle que la glycémie moyenne des 60 derniers jours s'établit à 2,20 g/l. 
En l'absence d'autre cause, ce décès semble compatible avec l'une des complications d'un coma diabétique acido-cétosique.

\section{Discussion}

Si l'EDTA constitue l'anticoagulant de choix, d'ailleurs recommandé, pour la détermination de $\mathrm{l}^{\prime} \mathrm{HbA}_{\mathrm{IC}}$, d'autres substances peuvent être utilisées. L'EDTA liquide utilisé en raison de ses propriétés protectrices de niembrane permet une meilleure conservation des globules rouges. L'héparinate de lithium pouvant donner des débris de stroma dans l'hémolysat n'est pas recommandé (selon le fabriquant du Diamat). Quant au fluorure, ce sel est un puissant agent hémolysant et n'autorise une bonne séparation de $1^{\prime} \mathrm{HbA}_{1 \mathrm{C}}$ de $\mathrm{l}^{\prime} \mathrm{HbA}_{0}$ que pendant une période moyenne de 3 mois lorsque les échantillons sont conservés à $+4^{\circ} \mathrm{C}$. Après 3 mois, nous constatons que la dénaturation de $\mathrm{l}^{\prime} \mathrm{Hb}$ aboutit à une production d'Hb dont la structure est modifiée et qui migre entre $1 \mathrm{HbA}_{0}$ et l'HbA ${ }_{1 C}$ rendant difficile ou impossible l'intégration de $l^{\prime} \mathrm{HbA}_{1 \mathrm{C}}(8)$. Sur le cadavre, les prélèvements sur tube "sec" sont préférables à ceux réalisés sur fluorure, pouvant être analysés pendant une période d'un an. En ce qui concerne les sujets vivants, les prélèvements réalisés sur fluorure et sur tube sec sont formellement contreindiqués. La congélation à $-20^{\circ} \mathrm{C}$ constitue vraisemblablement le plus mauvais moyen de conservation en raison de l'hémolyse des globules rouges qui se produit lors des chocs thermiques accompagnant la congélation et la décongélation. Il est possible de proposer par ordre de préférence décroissant les modalités de stockage suivants : tube EDTA $+4^{\circ} \mathrm{C}>$ tube "sec" $+4^{\circ} \mathrm{C}>$ tube hépariné $+4^{\circ} \mathrm{C}$. Quelque soit le type de prélèvement, la détermination de l' $\mathrm{HbA}_{1 \mathrm{C}}$ est possible pendant une période minimale de 3 mois lorsque les échantillons sont conservés à $+4^{\circ} \mathrm{C}$. Toute élévation de l' $\mathrm{HbA}_{1 \mathrm{C}}$, associée ¿ une acétonémie élevée permet d'attribuer à un diabète l'élévation de l'acétonémie. Lors de l'étude de conservation un certain nombre de dosages d' $\mathrm{HbA}_{1 \mathrm{C}}$ se sont révélés augmentés, dans tous les cas, la confirmation du diabète a pu être réalisée montrant que la conservation de l'HbA $1_{1 \mathrm{C}}$ semble bonne au cours du temps. Le facteur limitant l'interprétation des résultats est l'apparition d'un pic d'hémoglobine dénaturée $\mathrm{HbA}_{\text {Id }}$ qui migre dans les conditions retenues entre $\mathrm{l}^{\prime} \mathrm{HbA}_{0}$ et $\mathrm{l}^{\prime} \mathrm{HbA}_{1 \mathrm{C}}$ et qui rend impossible la détermination de l' $\mathrm{HbA}_{1 \mathrm{c}}$. Des conditions chromatographiques sensiblement modifiées pour de tels échantillons mériteraient d'être essayées. A défaut, l'emploi d'autres techniques séparatives comme l'électrophorèse ou l'isoélectrofocalisation peut constituer une bonne alternative. En ce qui concerne les cas médico-légaux, le dosage de l' $\mathrm{HbA}_{1 \mathrm{C}}$ apporte des informations déterminantes sur la glycémie moyenne des
60 derniers jours alors qu'aucun paramètre biochimique classique n'est interprétable, y compris la glycémie. Non seulement ce dosage complète utilement l'acétonémie, mais il permet comme dans le cas $\mathrm{N}^{\circ} 3$, en l'absence d'autre cause d'attribuer l'origine du décès à un coma diabétique, chez un sujet présentant une acétonémie et une l' $\mathrm{HbA}_{\mathrm{IC}}$ élevées. Les facteurs susceptibles d'affecter les résultats et/ou leur interprétation doivent être pris en compte : présence d'hémoglobine anormale en concentration importante en particulier.

\section{Conclusion}

L'hémoglobine glyquée ou l'HbA $\mathrm{A}_{1 \mathrm{C}} \mathrm{s}$ 'avère un complément extrêmement utile du dosage de l'acétonémie lorsque celle-ci est élevée. Elle peut contribuer à une meilleure connaissance des causes de la mort d'un sujet, en particulier pour tout décès inexpliqué. Après l'acétone, l'hémoglobine glyquée est le second paramètre biochimique dont l'interprétation est valide en post-mortem. Cette technique de grande routine biologique est facile à mettre en œuvre et ne nécessite qu'une prise d'essai extrêmement réduite.

\section{Références}

1. Thomsen J.L., Theilade P., Felby S., Nielsen E. Alcoholism and ketoacidosis. Forensic Sci. Int. 1993 ; $60: 3-4$

2. Thomsen J.L., Felby S., Theilade P., Nielsen E. Alcoholic ketoacidosis as a cause of death in forensic cases. Forensic Sci. Int. $1995 ; 75$ (2-3) : 163-71.

3. Pounder D.J., Stevenson R.J., Taylor K.K. Alcoholic ketoacidosis at autopsy. J. Forensic. Sci. $1998 ; 43$ (4) : 812-6.

4. Brinkmann B., Fechner G., Karger B., Du Chesne A. Ketoacidosis and lactic acidosis-frequent causes of death in chronic alcoholics ? Int. J. Legal Med. $1998 ; 111$ (3) : 115-9.

5. Goullé J.P., Lacroix C., Anagnostides J., Gharios G., Perdue-Legendre E., Rigaud J.P., Nouveau J. L'acétone : un marqueur biologique trop souvent négligé. Toxicorama. $1999 ; 11$ (4) : 265-9.

6. Logan B.K., Gullberg R.G., Elenbaas J.K. Isopropanol interference with breath alcohol analysis : a case report. J. Forensic Sci., $1994 ; 39$ (4) : $211-5$.

7. Nathan D.M., Singer D.E. et al. The clinical information value of the glycosylated hemoglobin assay. N. Eng. J. Med., $1984 ; 310: 341-6$.

8. Gillery P., Guillemin C., Delpech M. Hémoglobine glyquée : méthodes de dosage et problèmes de standardisation. Ann. Biol. Clin., 1994 ; 52 : 157-63.

9. Vassault A., Grafmeyer D., Naudin Cl., Dumont G., Bailly M., Henny J., Gerhardt M.F., Georges P. Protocole de validation de techniques. L'information scientifique du biologiste, $1985 ; 11: 5-51$. 